\title{
Estudio comparativo del proceso de secado de Eucalyptus grandis en dos secaderos solares ubicados en diferentes zonas y un secado natural a la intemperie durante el invierno
}

\author{
De Castro, R. ${ }^{(1)}$, Cárdenas, P. ${ }^{(1)}$, Venturino, A. ${ }^{(2)}$, Ono, A. ${ }^{(3)}$ \\ Contacto: rdecas@latu.org.uy \\ (1) Departamento de Proyectos Forestales, Laboratorio Tecnológico del Uruguay, (LATU) - (2) Departamento de Proyectos \\ Forestales (2004 - 2006), LATU - ${ }^{(3)}$ Departamento de Proyectos Forestales (2002 - 2006), LATU
}

Recibido: 8/9/2008 - Aprobado: 27/11/2008

\begin{abstract}
$\underline{\text { Resumen }}$
En la siguiente nota técnica se presentan los resultados de tres ensayos de secados de tablas de Eucalyptus grandis de $27 \mathrm{~mm}$ de espesor, realizados durante los meses de invierno. Dos de ellos fueron realizados en secaderos solares tipo semi invernadero (ubicados en Paysandú y en Montevideo), y uno fue realizado secando a la intemperie (en Montevideo).

Se registraron las condiciones de humedad relativa y temperatura del aire durante los tres ensayos y se compararon los resultados obtenidos. El secado solar realizado en Paysandú tuvo una duración de 95 días y se logró un contenido de humedad medio (CHM) final en base seca de 10,5 \%. El secado solar realizado en Montevideo duró 80 días y se llegó a un CHM final de 10,5 \%. EI secado a la intemperie realizado también en Montevideo tuvo lugar a lo largo de 80 días y se obtuvo un CHM final de $22,1 \%$. Se obtuvieron resultados similares para ambos secados solares, teniendo en cuenta las diferencias de diseño, de ubicación y de capacidad. Comparando los secados solares con el secado a la intemperie, los primeros llevaron la madera de verde (CHM de $105 \%$ aprox.) hasta un CHM de $22 \%$ en la mitad de tiempo. Según los resultados obtenidos, es posible reemplazar el secado a la intemperie por un secado solar y lograr así acortar los tiempos de secado a un bajo costo.

Palabras clave: Secado solar, secado a la intemperie, madera
\end{abstract}

\begin{abstract}
In the following report, the results of three drying runs made during winter with boards of Eucalyptus grandis $\mathbf{2 7} \mathbf{~ m m}$ thick are shown. Two of them took place in solar kilns, semi-greenhouse type (located in Montevideo and Paysandú), and the third took place outdoors (located in Montevideo).

The relative humidity and temperature conditions of the three drying runs were registered and the results obtained were compared. The solar drying in Paysandú lasted 95 days, and achieved a final average moisture content (AMC) of 10,5\% in dry basis. The other solar drying which took place in Montevideo lasted 80 days and achieved a final AMC of 10,5 \%. The outdoors drying process lasted 80 days in Montevideo and achieved a final AMC of 22,1\%.

Similar results were obtained for both solar drying processes, taking into consideration the differences in design, location and capacity. Comparing the solar drying with the ambient air drying process, drying time from green condition to $22 \%$ AMC in solar kilns was half time of outdoors drying. According to this result, it is possible to replace the outdoors drying process by a solar drying and thus shorten the drying time at a low cost.

Key words: Solar drying, outdoors drying, wood.
\end{abstract}

\section{Introducción}

La siguiente nota técnica forma parte de una serie de trabajos de investigación del Departamento de Proyectos Forestales del Laboratorio Tecnológico del Uruguay (LATU) que toman en consideración la importancia que tiene actualmente el uso de energías alternativas en procesos industriales como el secado solar de maderas.

La finalidad de este trabajo fue desarrollar tres experiencias de secado en paralelo para estudiar la adecuación y la optimización de programas de secado en hornos solares. Asimismo se buscó generar algunos conocimientos que permitieran implementar apropiadamente dicha tecnología.

Los objetivos propuestos para este trabajo fueron determinar curvas de secado, contenido de humedad $(\mathrm{CH})$ final alcanzado, tiempo requerido y calidad del producto seco (contracción y distribución de $\mathrm{CH}$ dentro de las tablas).
Para poder cumplir con los objetivos propuestos se utilizaron dos secaderos solares ubicados respectivamente en la zona norte y sur del país y una estiba que se secó por secado natural a la intemperie.

\section{Materiales y Métodos}

La madera provino de rodales de la zona de Piedras Coloradas, de árboles correspondientes a un primer raleo de 12 años de edad promedio. Las tablas utilizadas para este trabajo fueron de E. grandis de plantaciones de Forestal Caja Bancaria y presentaron un peso específico básico promedio de $0,46 \mathrm{~g} / \mathrm{cm}^{3}$.

Se relevó el ancho, espesor, largo y peso de las tablas verdes y luego de secadas. Las dimensiones iniciales de las tablas utilizadas por el LATU para los dos secados (solar e intemperie) fueron de $105 \mathrm{~mm}$ x $27 \mathrm{~mm}$ x $215 \mathrm{~cm}$. Las dimensiones iniciales de las tablas utilizadas 
por FCB fueron: la mitad de las tablas $142 \mathrm{~mm}$ x $27 \mathrm{~mm} \times 215 \mathrm{~cm}$; la otra mitad $107 \mathrm{~mm}$ x $27 \mathrm{~mm}$ x $215 \mathrm{~cm}$.

También se evaluó el gradiente de humedad interna de las tablas secadas al finalizar el secado. Para esto se tomaron probetas de las tablas y se determinó el $\mathrm{CH}$ en cinco posiciones.

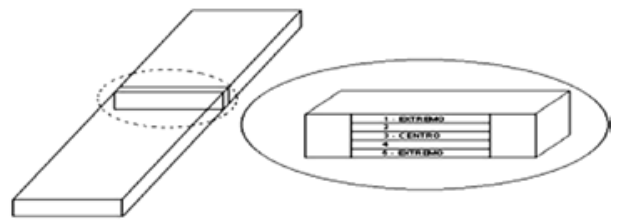

Figura 1. Croquis de probetas de gradiente de humedad (corte transversal).

\section{Metodología de trabajo en Forestal Caja Bancaria}

Dentro del horno se colocaron un total de ocho paquetes de tablas, con un volumen de aproximadamente $1 \mathrm{~m}^{3}$ cada uno:

Cuatro paquetes tenían tablas de dimensiones $142 \mathrm{~mm} \times 27 \mathrm{~mm} \times 215 \mathrm{~cm}$. Cuatro paquetes tenían tablas de dimensiones $107 \mathrm{~mm} \times 27 \mathrm{~mm} \mathrm{x}$ $215 \mathrm{~cm}$.

Las estibas se construyeron con separadores de $25 \mathrm{~mm}$ espaciados entre sí a $40 \mathrm{~cm}$ (cinco separadores por fila). Sobre los paquetes no se colocó peso, aunque algunos paquetes sí lo tuvieron, debido a que se colocó otro paquete sobre ellos.

Se tomaron tres muestras (testigos) para evaluar la evolución del contenido de humedad de la madera durante el secado. De cada muestra se extrajeron dos probetas para obtener el contenido de humedad inicial de cada testigo.

Se colocó un registrador de humedad y temperatura (TANDD thermo Recorder TR $72 \mathrm{~S}$ ) para tener registro de las condiciones del aire dentro del horno solar.

El seguimiento del contenido de humedad media de los testigos se realizó semanalmente por pesadas y estuvo a cargo del personal de Forestal Caja Bancaria. También se realizaron medidas de contracción en distintas etapas de la experiencia.

Una vez finalizado el secado se extrajeron tablas para determinar la distribución del $\mathrm{CH}$ final.

\section{Características del secador solar de Forestal Caja Bancaria}

Ubicación: Piedras Coloradas, departamento de Paysandú, cubierta transparente inclinada orientada hacia al Norte. Latitud $32^{\circ} 20^{\prime} 57^{\prime \prime} \mathrm{S}$, longitud $58^{\circ} 02^{\prime} 13^{\prime \prime} \mathrm{W}$, altitud $61.00 \mathrm{~m}$ s.n.m.

- Secador tipo semi-invernadero, aislación de espuma de poliestireno expandido (Espumaplast) entre tablas de madera de pino tratadas con CCA.

- Techo de poliéster reforzado con fibra de vidrio con superficie total $50 \mathrm{~m}^{2}: 28 \mathrm{~m}^{2}$ con una inclinación de $50^{\circ}$, y $22 \mathrm{~m}^{2}$ con una inclinación de $20^{\circ}$.

- Colector solar tipo plato (chapas de OSB negro). Los colectores son móviles.

Los colectores superiores se colocan apoyados sobre la pila de madera.

Las paredes internas del horno se encuentran pintadas de negro (actuando como colectores)

- Ventiladores: dos de $1 / 2 \mathrm{HP}$ cada uno (50 cm de diámetro) con variadores de velocidad.
- Ventilas: dos al frente, con dos extractores en la parte superior.

- Piso: losa de hormigón $58,7 \mathrm{~m} 2$ y $30 \mathrm{~cm}$ de alto.

- Capacidad: $8 \mathrm{~m}^{3}$ de madera.

- Área de colector por volumen de capacidad: $6,25 \mathrm{~m}^{2} / \mathrm{m}^{3}$

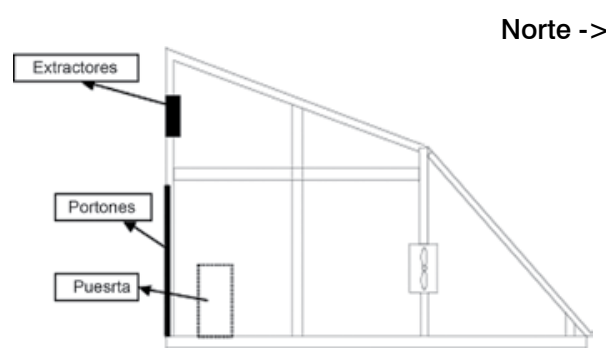

Figura 2. Secador solar de FCB (vista lateral).

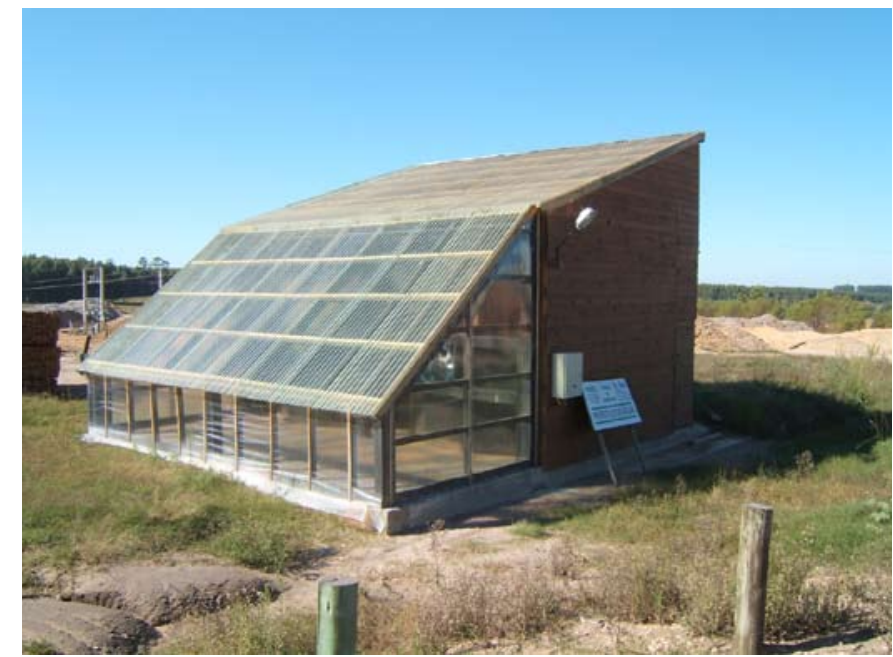

Figura 3. Secador solar de Forestal Caja Bancaria.

\section{Metodología de trabajo en el LATU}

Con las tablas a secar se armaron dos estibas, una para su secado en el horno solar y otra para secado natural a la intemperie, protegida solo por una chapa en la parte superior.

Las estibas se construyeron con separadores de $28 \mathrm{~mm}$ x $28 \mathrm{~mm}$ colocados cada $40 \mathrm{~cm}$ (cinco separadores por fila). Sobre cada una de ellas se colocó un peso de $700 \mathrm{~kg}$ a razón de $350 \mathrm{Kg} / \mathrm{m} 2$.

De ambas estibas se extrajeron ocho muestras (cuatro para la estiba del horno solar y cuatro para la estiba de secado a la intemperie) para el monitoreo del CHM durante el secado. Para la obtención del CHM de las muestras se hizo el mismo procedimiento que en FCB.

Se colocó un registrador (TANDD thermo Recorder TR 72 S) de humedad y temperatura en el horno solar y otro en la estiba a la intemperie para evaluar ambas condiciones del aire de secado.

El seguimiento del CHM de los testigos se realizó por pesadas semanales por parte del LATU. También se realizaron medidas de contracción en distintas etapas de la experiencia.

Una vez finalizado el secado se extrajeron tablas para estudiar la distribución del $\mathrm{CH}$ final.

\section{Características del secador solar del LATU}

- Ubicación: departamento de Montevideo, latitud 34 54'43" S, longitud $56^{\circ} 04^{\prime} 28^{\prime}$ ' W, altitud $26.7 \mathrm{~m}$ s.n.m.

- Horno tipo semi-invernadero con aislación de poliestireno expandido entre tableros de contrachapados fenólicos.

- Techo de policarbonato $24 \mathrm{~m}^{2}$, inclinación $36^{\circ}$. 
- Colector solar tipo plato y tipo caja, de contrachapado fenólico pintado de negro.

- Ventiladores: dos de $1 / 2 \mathrm{HP}$ cada uno (60 cm de diámetro) con variadores de velocidad.

- Ventilas: dos al frente del horno.

- Piso: losa de hormigón $25 \mathrm{~m}^{2}$ y $15 \mathrm{~cm}$ de alto.

- Capacidad: $2,5 \mathrm{~m}^{3}$ de madera.

- Área de colector por volumen de capacidad: $9,6 \mathrm{~m}^{2} / \mathrm{m}^{3}$

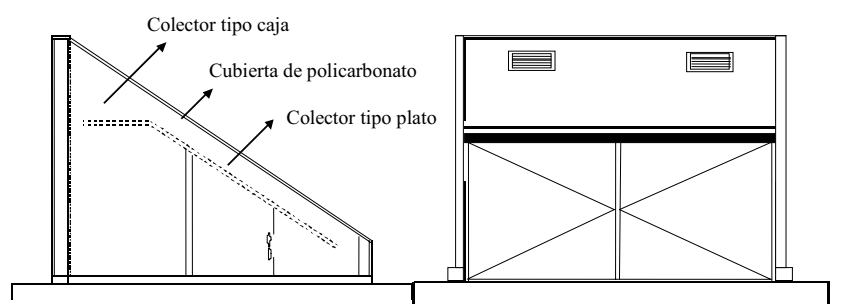

Figura 4. Esquema de horno solar del LATU.

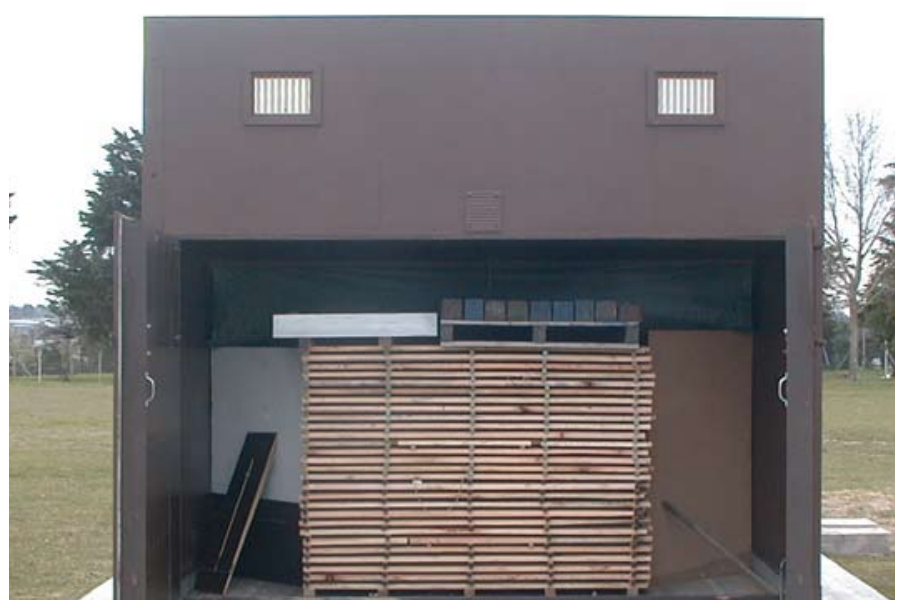

Figura 5. Secador solar de LATU con carga de E. grandis.

\section{Resumen de los costos de los secadores solares}

El costo de construcción por metro cúbico de capacidad del secador solar de FCB fue $600 \mathrm{USD} / \mathrm{m}^{3}$ aproximadamente, para $8 \mathrm{~m}^{3}$. Para el horno solar del LATU fue de $2.086 \mathrm{USD} / \mathrm{m}^{3}$, para una capacidad de $2,5 \mathrm{~m}^{3}$. Es importante resaltar que fueron construidos con diferentes fines. Si bien ambos hornos son a escala piloto, el horno de FCB se acerca más a lo que sería un horno a escala industrial, mientras que el horno solar del LATU se construyó con fines de investigación.

\section{Resultados}

\section{SECADO SOLAR DE E. grandis EN FORESTAL CAJA BANCARIA}

\section{Monitoreo de temperatura y de humedad relativa}

El Gráfico 1 resume las condiciones del aire dentro del horno solar durante el secado. La temperatura máxima registrada fue de $45,9^{\circ} \mathrm{C}$ y el mínimo porcentaje de humedad relativa (HR) fue de $22 \%$.

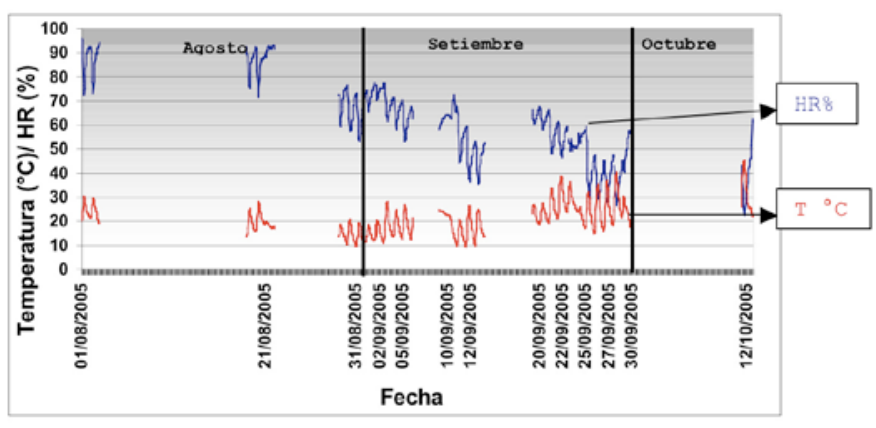

Gráfico 1. Condiciones de temperatura y humedad relativa del aire dentro del horno solar.

\section{Contenido de humedad de equilibrio de la madera durante el período de secado}

Los datos registrados muestran la tendencia a la disminución del CHE de la madera dentro del secador a medida que avanza el secado (Gráfico 2).

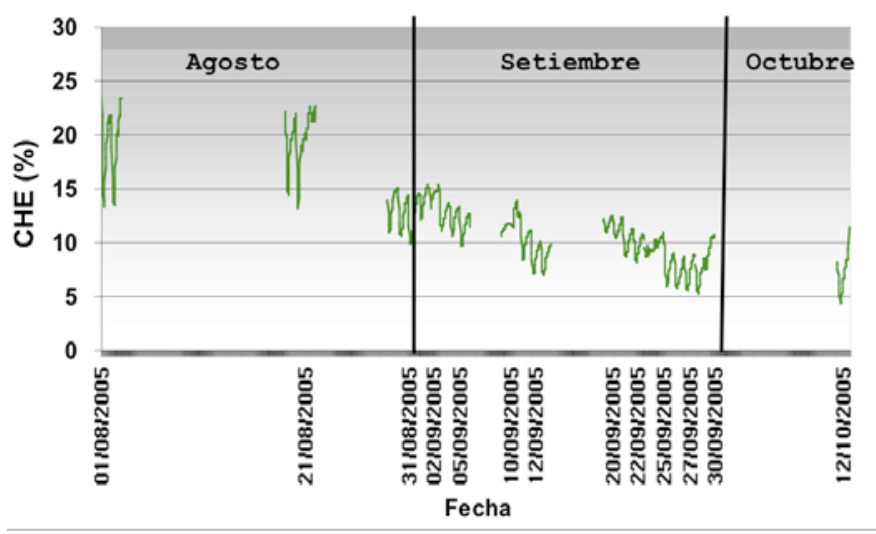

Gráfico 2. Contenido de humedad de equilibrio dentro de la madera del horno solar.

El valor mínimo registrado para el CHE fue de 4,3\%.

\section{Evolución del contenido de humedad medio de la madera}

El secado llevó un período de 95 días y se llegó a un CHM final de $10,5 \%$ en base seca (Tabla 1 ). 


\begin{tabular}{|l|c|c|}
\hline & CHM inicial & CHM final \\
\hline Promedio (\%) & 103 & 10,5 \\
\hline Desviación Estándar & 17,6 & 0,8 \\
\hline C.V. (\%) & 17 & 8 \\
\hline
\end{tabular}

Tabla 1. Contenido de humedad de la madera.

El promedio de CHM llegó a 12 \% en 78 días.

En el Gráfico 3 se puede observar la evolución del secado, donde se puede notar no solo la disminución del CHM, sino también la disminución de la variabilidad entre tablas.

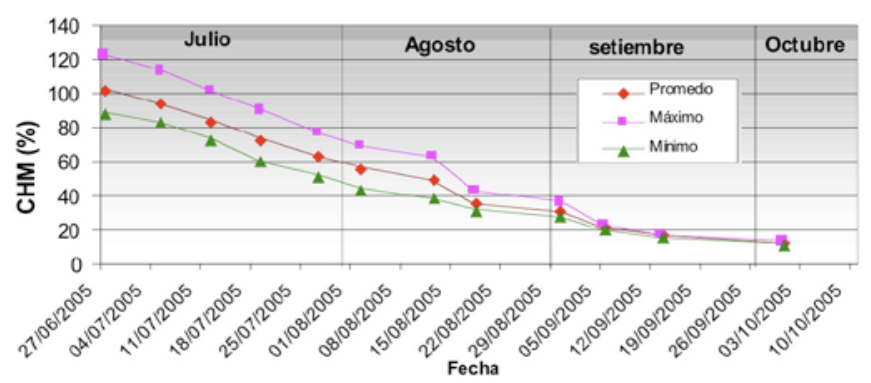

Gráfico 3. Curva de secado en horno solar FCB.

\section{Variaciones del contenido de humedad en el interior de la madera}

En el Gráfico 4 se describe la distribución de la humedad interna para distintas posiciones en el interior de la tabla de madera. Se hicieron determinaciones en cinco tablas distribuidas en el ancho de la estiba. Excepto la muestra que se encontró con valores de CHM superior al $12 \%$, la variación del CHM entre el centro de la tabla y sus extremos fue aceptable y está dentro de 1,3\% de variación.

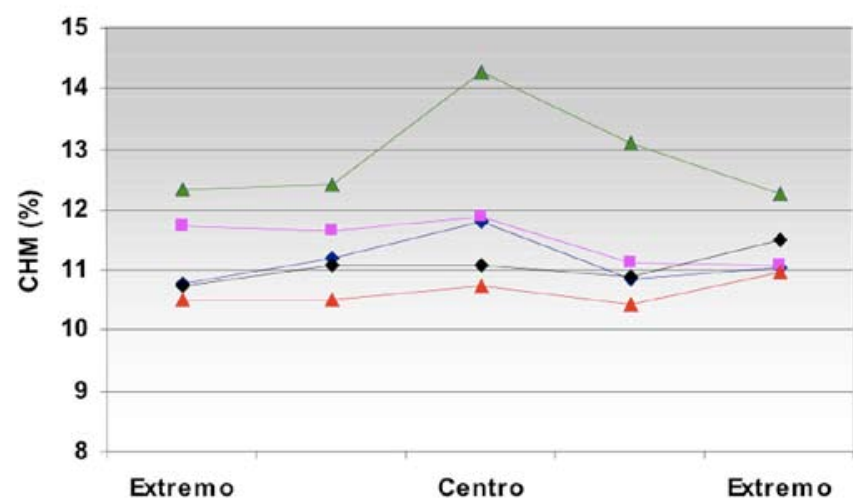

Gráfico 4. Variación de la humedad interna para distintas posiciones en el interior de cinco tablas.

\section{Contracción volumétrica de las tablas}

En la Tabla 2 se resume la contracción volumétrica que se produjo para una variación del CHM desde un valor inicial de $103 \%$ hasta un CHM final (10,5\%) para seis tablas de $142 \mathrm{~mm} \times 27 \mathrm{~mm} \times 215 \mathrm{~cm}$ y para nueve tablas de $107 \mathrm{~mm} \times 28 \mathrm{~mm} \times 215 \mathrm{~cm}$.

\begin{tabular}{|c|c|c|c|}
\hline & \multicolumn{3}{|c|}{ Contracción (\%) } \\
\hline Ancho de tablas & Ancho & Espesor & Volumétrica \\
\hline $142 \mathrm{~mm}$ & 7,6 & 5,2 & 12,8 \\
\hline $107 \mathrm{~mm}$ & 6,8 & 3,8 & 10,6 \\
\hline
\end{tabular}

Tabla 2. Contracción luego del secado.

\section{SECADO SOLAR Y SECADO NATURAL A LA INTEMPERIE DE E. Grandis EN EL LATU}

\section{Condiciones de humedad relativa ambiente y dentro del horno solar y a la intemperie}

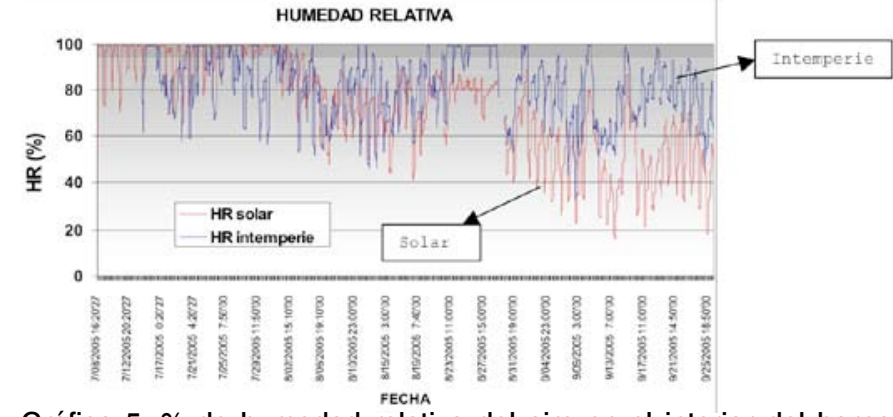

Gráfico 5. \% de humedad relativa del aire en el interior del horno solar y a la intemperie.

La humedad relativa del aire dentro del horno no tuvo variaciones bruscas durante las primeras semanas, con valores altos que permitían un secado progresivo. Cuando el contenido de humedad de la madera llegó aproximadamente a $40 \%$, la humedad relativa del aire dentro del secador comenzó a bajar, llegando a niveles que resultaron en un gradiente que mantiene el potencial de secado.

\section{Condiciones de temperatura del ambiente y dentro del horno solar y la intemperie}

La temperatura dentro del horno solar fue en promedio $7,4{ }^{\circ} \mathrm{C}$ superior a la temperatura del ambiente exterior (Gráfico 6).

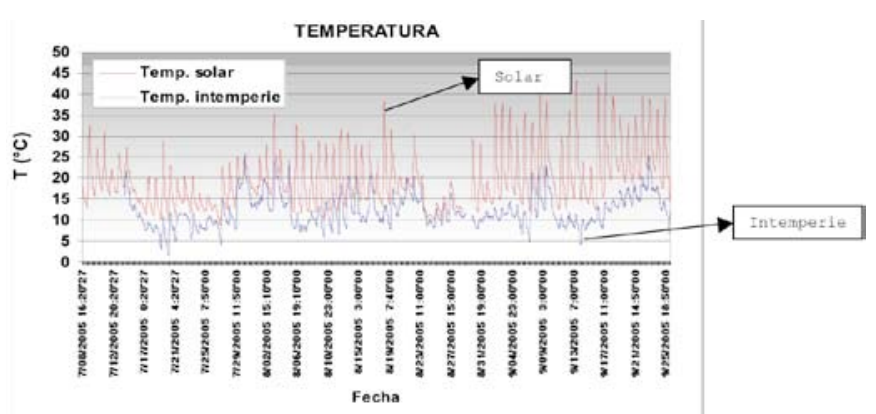

Gráfico 6. Temperatura ambiente y en el interior del horno solar y a la intemperie 


\begin{tabular}{|c|c|c|c|c|c|c|}
\hline & \multicolumn{5}{|c|}{ Temperatura del aire $\left({ }^{\circ} \mathrm{C}\right)$} \\
\hline & \multicolumn{2}{|c|}{ Julio } & \multicolumn{2}{|c|}{ Agosto } & \multicolumn{2}{c|}{ Setiembre } \\
\hline Fecha & Ambiente & $\begin{array}{c}\text { Horno } \\
\text { solar }\end{array}$ & Ambiente & $\begin{array}{c}\text { Horno } \\
\text { Solar }\end{array}$ & Ambiente & $\begin{array}{c}\text { Horno } \\
\text { solar }\end{array}$ \\
\hline Promedio & 10,8 & 16,3 & 12,6 & 18,4 & 12,7 & 23,3 \\
\hline Máxima & 25,8 & 28,7 & 24,8 & 38,2 & 25,4 & 45,9 \\
\hline Mínima & 1,8 & 8,7 & 5,5 & 10,3 & 4,1 & 12,1 \\
\hline
\end{tabular}

Tabla 3. Temperaturas promedio, máxima y mínima, del aire en el interior del horno solar y a la intemperie según el mes del año.

\section{Contenido de humedad de equilibrio de la madera durante el periodo de secado}

El comportamiento del CHE de la madera fue similar al de la humedad relativa del aire en el exterior (Gráfico 7).

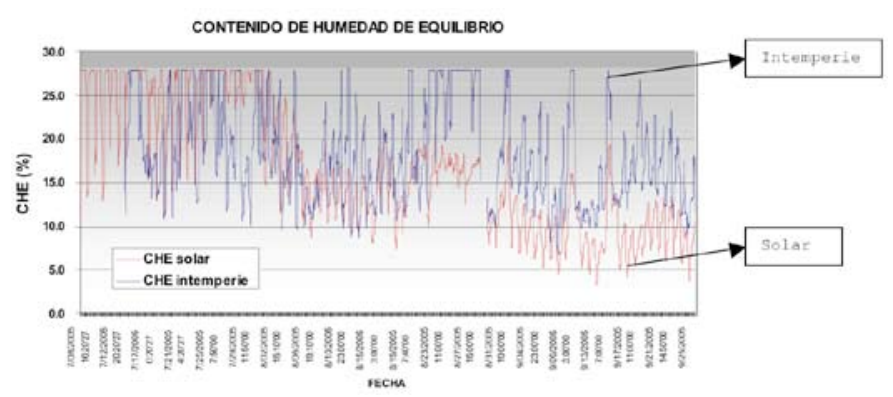

Gráfico 7. Contenido de humedad de equilibrio de la madera en el interior del horno solar y a la intemperie.

\begin{tabular}{|c|c|c|c|c|c|c|}
\hline & \multicolumn{5}{|c|}{$\begin{array}{r}\text { Contenido de humedad de equilibrio } \\
\text { de la madera (\%) }\end{array}$} \\
\hline & \multicolumn{2}{|c|}{ Julio } & \multicolumn{2}{c|}{ Agosto } & \multicolumn{2}{c|}{ Setiembre } \\
\hline Fecha & Ambiente & $\begin{array}{r}\text { Horno } \\
\text { Solar }\end{array}$ & Ambiente & $\begin{array}{r}\text { Horno } \\
\text { Solar }\end{array}$ & Ambiente & $\begin{array}{c}\text { Horno } \\
\text { Solar }\end{array}$ \\
\hline Promedio & 21,1 & 25,8 & 18,9 & 15,4 & 16,2 & 9,7 \\
\hline Máxima & 28 & 28 & 28 & 27.8 & 28 & 20,2 \\
\hline Mínima & 10,1 & 11,1 & 8,6 & 7,4 & 6,6 & 3,3 \\
\hline
\end{tabular}

Tabla 4. Contenido de humedad de equilibrio promedio, máximo y mínimo, de la madera según el mes del año.

\section{Evolución del contenido \\ de humedad medio de la madera}

El secado de E. grandis fue llevado a cabo en 80 días, como se muestra en el Gráfico 8. Se partió de la madera verde con un contenido de humedad medio de $107 \%$ y se secó hasta $10,5 \%$ en base seca (Tabla 5).
Contenidos de humedad inicial y final de la madera en el secado solar

\begin{tabular}{|l|c|c|}
\hline & CHM inicial & CHM final \\
\hline Promedio (\%) & 107,3 & 10,5 \\
\hline Desviación Estándar & 18,5 & 0,3 \\
\hline C.V. (\%) & 17,2 & 2,7 \\
\hline
\end{tabular}

Tabla 5. Contenido de humedad medio de la madera del secado solar.

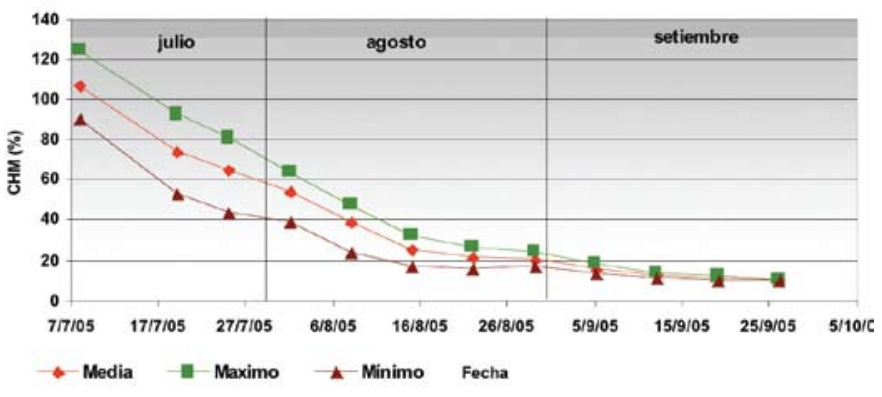

Gráfico 8. Curva de secado solar de tablas en el horno solar del LATU.

\section{Variaciones del contenido de humedad en el interior de la madera}

El Gráfico 9 describe la distribución del gradiente de humedad interna para las cuatro tablas testigo.

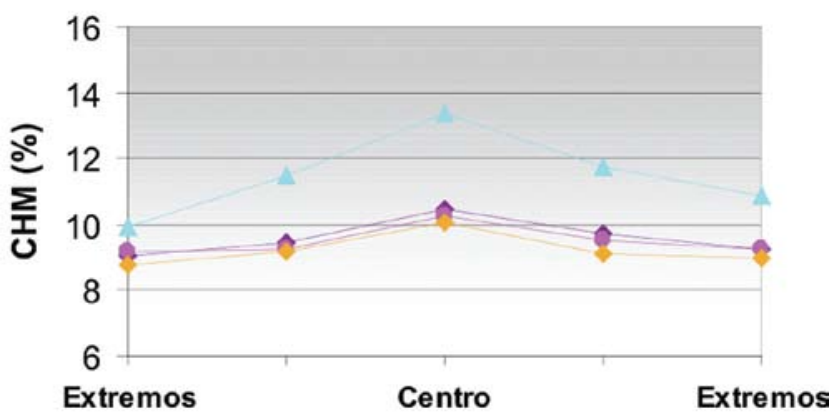

Gráfico 9: Variación de la humedad interna para distintas posiciones en el interior de las tablas testigo.

Excepto una tabla que se encontró con valores de CHM superior al $12 \%$, las restantes presentaron gradientes aceptables entre el centro y sus extremos, dentro del $1 \%$ de variación.

\section{Contracción volumétrica de las tablas}

La contracción volumétrica que se produjo durante el secado desde la condición verde (CHM $103 \%$ ) hasta un CHM de $11 \%$ se detalla en el Tabla 6.

\begin{tabular}{|c|c|c|c|}
\hline & \multicolumn{3}{|c|}{ Contracción (\%) } \\
\hline & Ancho & Espesor & Volumétrica \\
\hline Promedio (\%) & 6,4 & 4,7 & 11,1 \\
\hline
\end{tabular}

Tabla 6. Contracción volumétrica de tablas. 


\section{Contenidos de humedad media inicial y final de la madera del secado a la intemperie}

El secado a la intemperie se inició y finalizó al mismo tiempo que el secado solar. El período de secado fue de 80 días, llegando a un CHM final de $22,1 \%$ (Tabla 7).

\begin{tabular}{|l|c|c|}
\hline & CHM inicial & CHM final \\
\hline Promedio (\%) & 105,4 & 22,1 \\
\hline Desviación estándar & 18,2 & 2,9 \\
\hline C.V. (\%) & 17,3 & 13,3 \\
\hline
\end{tabular}

Tabla 7. Contenido de humedad de la madera estibada a la intemperie.

\section{Comparación de secado solar y secado a la intemperie en el LATU}

Como se observa en el Gráfico 10, a partir de un CHM de $60 \%$ se comienza a ver una ventaja del secado solar respecto al secado a la intemperie. El secado solar permite alcanzar un CHM en la madera que es la mitad del CHM que se logra en el secado a la intemperie para un mismo período de 80 días de secado.

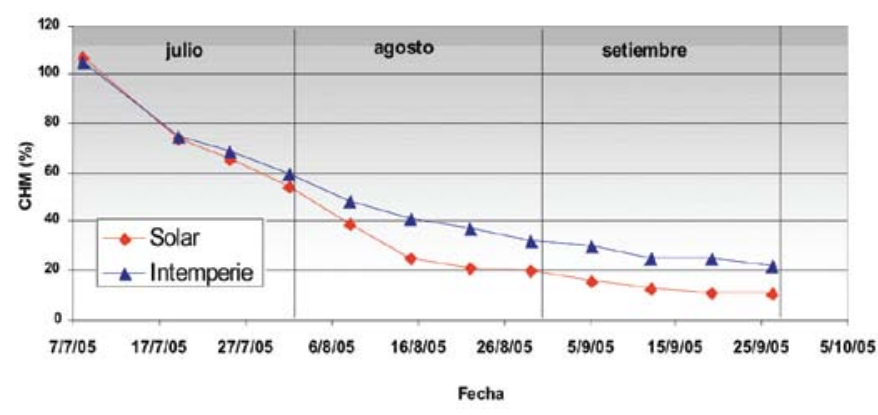

Gráfico 10. Curvas del secado por secado solar y por secado natural a la intemperie para tablas de $E$. grandis en el LATU.

\section{Resultados de secado solar en hornos de FCB y LATU}

En el horno solar del LATU se obtuvo un secado un poco más rápido que el del horno de FCB. Se deben tomar en cuenta las diferencias en diseño, capacidad y ubicación geográfica de ambos hornos (Gráfico 11). El horno solar del LATU tiene casi un $50 \%$ más de superficie colectora por $\mathrm{m}^{3}$ de capacidad con respecto al horno solar de FCB.

En las etapas finales se puede observar que prácticamente no hay diferencias entre el secador del LATU y el de FCB.

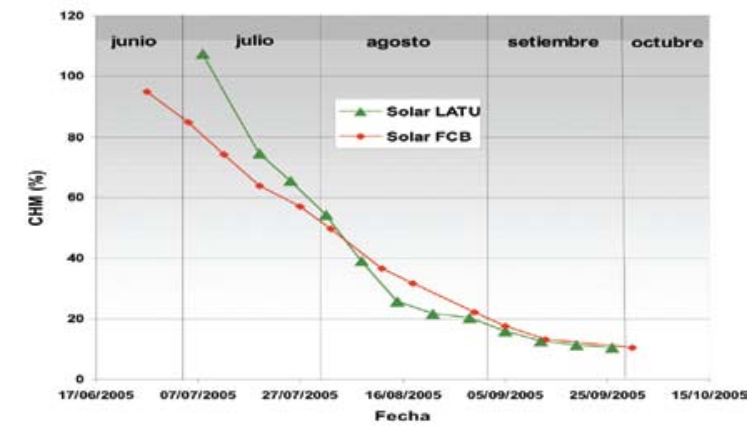

Gráfico 11. Curvas de secado solar en FCB y LATU.

\section{Conclusiones}

Se obtuvieron resultados de secado similares para ambos secadores solares, teniendo en cuenta las diferencias en diseño, ubicación y capacidad. Considerando que el secado se desarrolló de julio a setiembre, cuando las condiciones de radiación solar no son las óptimas, el resultado fue bueno, con valores de CHM final por debajo del $12 \%$ en tres meses, partiendo de madera en condición verde (CHM aprox. $105 \%$ ).

Dentro del secador solar hay mejores condiciones de secado que a la intemperie. Se logran una mayor velocidad de secado y condiciones controladas que producen menos defectos, como grietas y rajaduras.

Comparado con un secado a la intemperie durante la misma época del año, el secador solar llevó a la madera desde condición verde hasta $22 \%$ de $\mathrm{CH}$ en la mitad de tiempo. Por otra parte, un secado a la intemperie no permite lograr un CHM de madera por debajo del CHE dado por las condiciones de intemperie (14-18\%).

En base a los resultados obtenidos el secado solar, se presenta como una alternativa con mejores resultados que el secado natural a la intemperie. Los secadores solares para madera resultan una tecnología posible: su construcción tiene costos razonables (aproximadamente $600 \mathrm{USD} / \mathrm{m}^{3}$ ) que incluso pueden abaratarse con materiales disponibles en el aserradero, y costos de operación y mantenimiento mínimos (el costo de operación se limita a la alimentación eléctrica de los forzadores).

El secado solar es una alternativa que puede reemplazar el secado a la intemperie que se lleva a cabo antes del secado convencional en la estación estudiada (invierno).

Se podría utilizar la combinación de secado solar /secado convencional, logrando así menores tiempos de secado que los requeridos por la combinación de secado intemperie /secado convencional.

\section{Reconocimientos}

Los autores desean expresar su agradecimiento a Forestal Caja Bancaria, en especial, a las siguientes personas: Ing. Horacio Arévalo, Ing. Waldemar Annunziatto e Ing. Carlos Sarazola, quienes hicieron posible este trabajo.

\section{Referencias}

- ONO, Andrés. Secado solar de eucalyptus camaldulensis de $40 \mathrm{~mm}$ de espesor. Montevideo: LATU, 2006. (Nota Técnica; 7)

- ONO, Andrés; PÉREZ DEL CASTILLO, Álvaro; OHTA, Sadaaki. Diferentes etapas del proceso de secado. Montevideo: LATU, 2003. (Nota Técnica; 1)

- ONO, Andrés; VENTURINO, Alejandro. Secado solar de eucalipto colorado de $40 \mathrm{~mm}$ de espesor. Montevideo: LATU, 2006. (Nota Técnica; 6)

- ONO, Andrés; VENTURINO, Alejandro. Secado solar para madera: condiciones operativas. Montevideo: LATU, 2005. (Nota Técnica; 5)

- PÉREZ FAVARO, Álvaro; SAVER LEITE, Cyntya. Secado solar de tablas de eucalyptus y pinos. Montevideo: Facultad de Agronomía, 1999. 